\title{
Resonance-Based Procedure for Locating Failed Luminaires in AGL Systems
}

\author{
Daniel Vidal, Lluís Monjo, Luis Sainz
}

\begin{abstract}
Aeronautical ground lighting (AGL) systems provide a visual reference for aircraft during airport operations. Fast detection and location of failed luminaires is an important safety concern in AGL systems. In the event of luminaire failure, AGL transformers introduce harmonic currents. Resonances can increase harmonics, worsening the problem. This paper presents analytical expressions for determining resonance frequencies in AGL systems, as well as a simple procedure based on these expressions for locating failed luminaires from measurements.

Index Terms - AGL systems, failed luminaire location, resonance, harmonics, power quality.
\end{abstract}

\section{INTRODUCTION}

A eronautical ground lighting (AGL) systems assist aircraft pilots during approach, landing and taxiing [1]. These systems are 1 to $10 \mathrm{~km}$ single-phase series circuits where constant current regulators (CCRs) feed luminaires through transformers with voltages up to $5 \mathrm{kV}$ [1]-[10]. CCRs are variable voltage sources with standard power ratings of 4, 7.5, $10,15,20,25,30,50$ and $70 \mathrm{kVA}$ and a typical current rating of $6.6 \mathrm{~A}$. They provide an adjustable rms current according to luminaire brightness requirements and ensure the current rating even in case of luminaire failure [4], [7], [8]. Luminaires are visual aids for pilots providing five discrete brightness steps according to rms current values $(6.6,5.2,4.1,3.4$ and $2.8 \mathrm{~A}$ or 20.0, 15.8, 12.4, 10.3 and 8.5 A) [1], [2], [4], [11]. Their number ranges from 10 up to 300 in small and large AGL systems, respectively. AGL transformers isolate luminaires from the high operating voltages of the CCR primary circuit and ensure series circuit continuity even in the event of luminaire failure. According to the safety restrictions of the International Civil Aviation Organization and the Federal Aviation Agency, luminaire failure is an important issue in AGL systems, with fast detection and location of failed luminaires posing a challenge in terms of cost-saving maintenance and safe operating policies. Computer-controlled monitoring of AGL systems is suggested in the literature for checking the status of AGL systems (including failed luminaires) [3] - [6]. Power line communication (PLC)

This research was carried out with the support of the Aena Aeropuertos S.A Reus Airport (Spain).

D. Vidal is with the A.A.S.A. Airport of Reus, Spain (e-mail: dvlombarte@aena.es). Ll. Monjo is with the Department of Industrial Systems Engineering and Design-Electrical Engineering Area, University Jaume I, Spain (e-mail: 1monjo@uji.es). L. Sainz is with the Department of Electrical Engineering, ETSEIB-UPC, Av. Diagonal 647, 08028 Barcelona, Spain (email: sainz@ee.upc.es). systems between luminaires and CCRs are an attractive solution for AGL system monitoring [3], [5], [6]. However, this technology is still not sufficiently developed for deployment. For this reason, in many AGL systems (particularly, domestic AGL systems), failed luminaires are detected in-situ, leading to high maintenance costs [6]. In addition to the above safety issues, in the event of luminaire failure, transformers work in saturation when maintaining series circuit continuity, modifying CCR operating conditions and introducing harmonic currents into the AGL system, which damages power quality [8], [12], [13]. Resonances can arise in AGL systems because they have both inductive CCR and capacitive cable characteristics. Harmonic current emissions of AGL saturated transformers are amplified around resonance frequencies in case of luminaire failure, leading to increased voltage distortion [14]. The characterization of these resonances at failed luminaire terminals is necessary to predict and avoid these power quality problems. Resonance conditions can be analyzed from the AGL system equivalent circuit and frequency scan simulations [14]. However, analytical expressions are much more effective in characterizing resonance frequencies as a function of system parameters in order to further investigate their influence.

This paper studies the resonance problem in AGL systems and presents analytical expressions for characterizing resonance frequencies. A procedure for locating failed luminaires using these expressions and resonance frequency measurements is also presented. The study is supported by field measurements of AGL systems at Reus airport (Catalunya, Spain), where two circuits of 68 and 80 luminaires

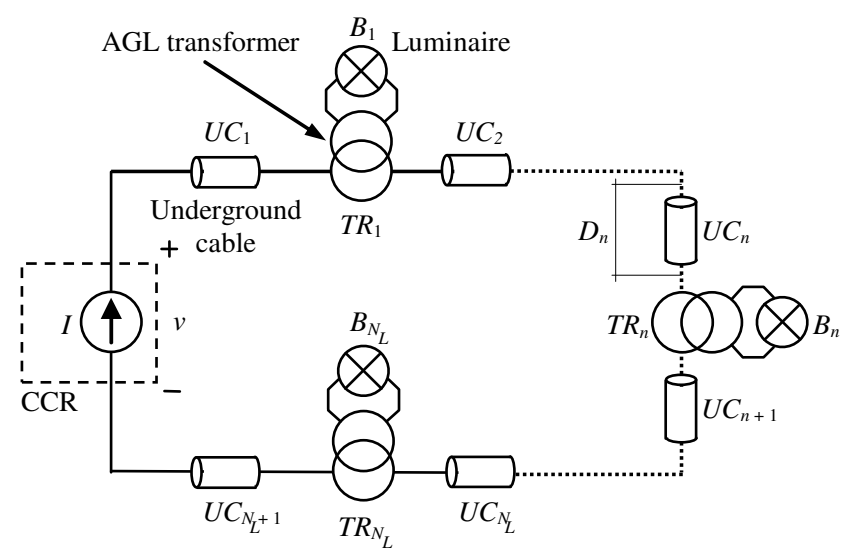

Fig. 1. AGL system. 
were tested in different operating conditions. Matlab/Simulink simulations [15] with the AGL system equivalent circuit in [14] are also used.

\section{AGL SYSTEMS}

AGL systems require expensive maintenance and conservation work and pose power quality concerns mainly derived from luminaire failure [5]. For this reason, it is necessary to study and predict AGL system behavior using an AGL system equivalent circuit.

\section{A. AGL System Description}

AGL systems are series circuits of copper insulated underground cables where CCRs supply $N_{L}$ luminaires through transformers with adjustable rms current $I$. Fig. 1 shows the basic circuit of these systems. As can be seen, the AGL transformers separate CCRs and luminaires into primary and secondary circuits, ensuring series circuit continuity and isolating luminaires from the primary circuit operating voltage, which can reach up to $5 \mathrm{kV} \mathrm{[1]-[8].} \mathrm{In} \mathrm{the} \mathrm{event} \mathrm{of} \mathrm{luminaire}$ failure, the transformers maintain circuit continuity but work in saturation because they operate in open circuit at series circuit rated current. This introduces harmonic currents into the AGL system, modifying CCR operating conditions and reducing AGL system power quality [14]. The presence of resonances in the AGL system can increase voltage and current distortions worsening the problem. The characterization of these resonances at failed luminaire terminals is necessary to analyze these power quality problems. This also allows failed luminaires to be located, as is presented in Section IV and applied in Section V.

\section{B. AGL System Equivalent Circuit}

AGL system behavior (in particular, AGL system resonances) can be studied from the AGL system equivalent circuit in Fig. 2 [14]. CCRs are modeled as a controlled sinusoidal voltage source of fundamental frequency $f_{1}$ in series

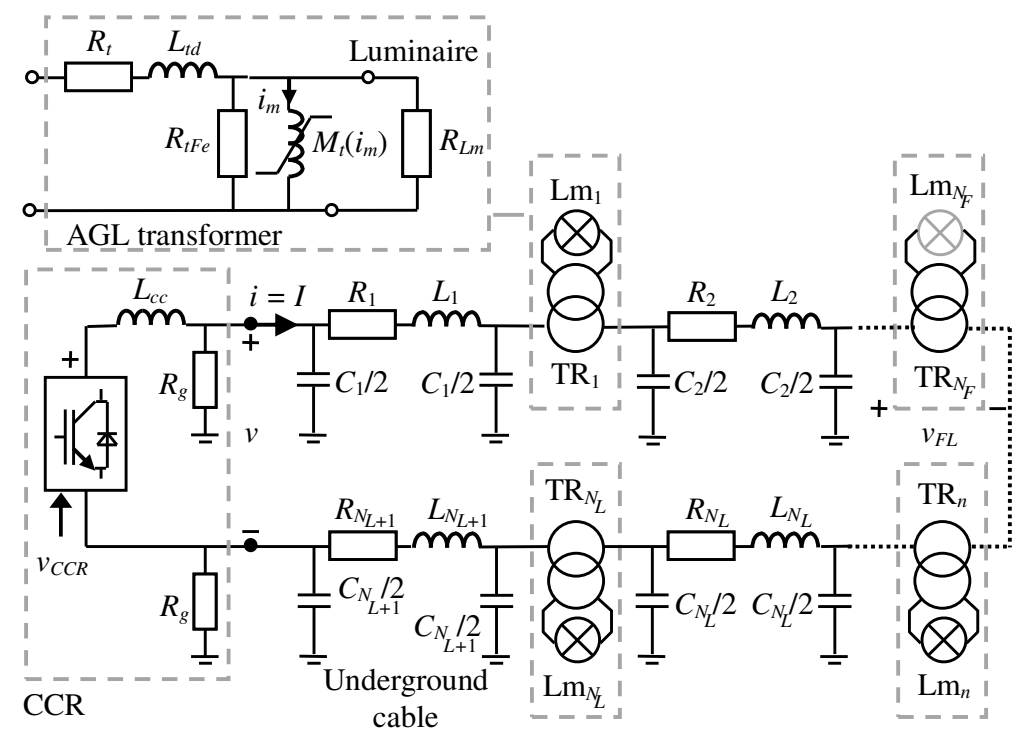

with the short-circuit inductance $L_{c c}$ of the CCR power transformer. Ground connected resistances $R_{g}(\approx \mathrm{M} \Omega)$ model earth fault detectors and lighting arrestors installed at CCR output terminals. AGL system cables are modeled with a concentrated parameter $\pi$-equivalent circuit because their length is typically smaller than $10 \mathrm{~km}$ and the shortest wavelength used in common AGL system operating conditions with frequencies below $2.5 \mathrm{kHz}$ is approximately greater than $40 \mathrm{~km}$. Cable conductance is neglected and the concentrated parameters $R_{n}, L_{n}$ and $C_{n}$ are obtained from the distributed parameters $R_{x}, L_{x}$ and $C_{x}$ and length $D_{n}$ of the cable as

$R_{n}=R_{x} \cdot D_{n} \quad L_{n}=L_{x} \cdot D_{n} \quad C_{n}=C_{x} \cdot D_{n} \quad\left(n=1, \ldots N_{L}+1\right) .(1)$

AGL transformers are characterized with the model proposed in [13], where $R_{t}$ and $L_{t d}$ are the winding resistance and constant leakage inductance, $R_{t F e}$ is the core loss resistance and $M_{t}\left(i_{m}\right)$ is the core non-linear magnetizing inductance, which depends on the magnetizing current $i_{m}$. AGL system luminaires are usually halogen lamps modeled as a resistance $R_{L m}$ [5], [8]. This resistance can be accurately calculated from the luminaire active power and current rating of the AGL system highest brightness step (i.e., $R_{L m}=P_{L m} / I_{L m}$, with $I_{L m}=6.6 \mathrm{~A}$ ). The luminaire $\mathrm{Lm}_{N F}$ in grey (see Fig. 2) represents the failed luminaire, whose transformer $\mathrm{TR}_{N F}$ works in saturation, introducing harmonic currents into the AGL system.

\section{AGL SYSTEM HARMONIC ANALYSIS}

The harmonic behavior of the passive set "observed" from the failed luminaire $\mathrm{Lm}_{N F}$ is studied in Fig. 3(a) to determine its harmonic response and resonance frequencies. Note that the CCR sinusoidal voltage source is short-circuited in the AGL harmonic equivalent circuit while the saturated consumed current of the failed luminaire $\operatorname{Lm}_{N F}$ is represented by the harmonic current source $I_{F L, k}$. The equivalent impedance $Z_{E q k}$ at the failed luminaire terminals must be analytically determined to characterize the resonance frequencies. As the

Fig. 2. AGL system equivalent circuit. 


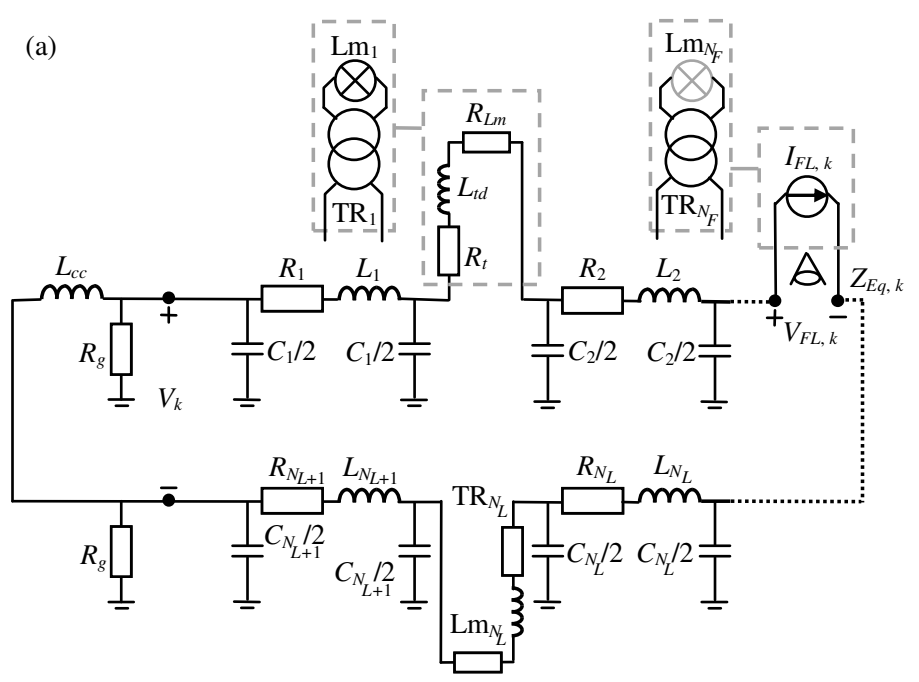

(b)

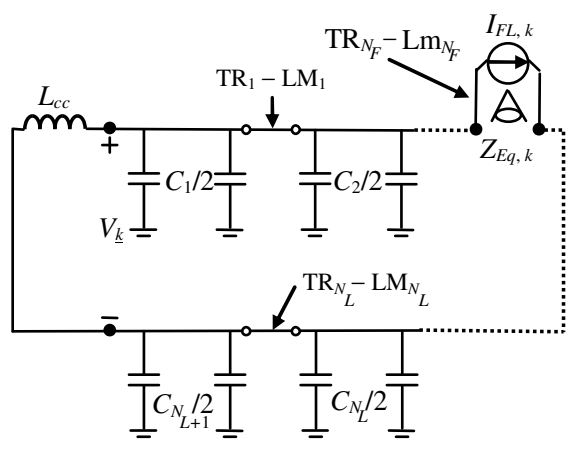

(c)

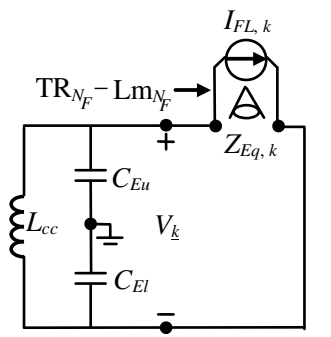

Fig. 3. AGL system harmonic equivalent circuit. (a) Complete circuit, (b) Simplified circuit and (c) Reduced circuit.

expression of this impedance is difficult to obtain with the circuit in Fig. 3(a), the two approximations below (validated in Section V) are made to work with a simple equivalent circuit and obtain accurate enough expressions of the resonance frequencies.

- The ground connected resistances $R_{g}$ are neglected because they are high value resistances $(\approx \mathrm{M} \Omega)$ in parallel with the CCR short-circuit reactance $X_{c c}=L_{c c} 2 \pi f_{1}$.

- The cable longitudinal impedance $R_{n}+j \omega L_{n}$, the transformer winding impedance $R_{t}+j \omega L_{t d}$ and the luminaire resistance $R_{L m}$ are also neglected because they are usually smaller than the CCR short-circuit reactance $X_{c c}$, and therefore the circuit harmonic voltage drop mainly occurs across this reactance.

Considering the above approximations, the AGL harmonic equivalent circuit in Fig. 3(a) can be simplified to the equivalent circuit in Fig. 3(b), which is reduced to the circuit in Fig. 3(c) by associating in parallel the capacitors before and after the failed luminaire:

$$
C_{E u}=\sum_{n=1}^{N_{F}} C_{n}=C_{x} D_{F} \quad C_{E l}=\sum_{n=N_{F}+1}^{N_{L}+1} C_{n}=C_{x}\left(D_{T}-D_{F}\right),
$$

where $D_{F}$ and $D_{T}$ are the length of the cables before of the failed luminaire and the total length of the AGL system cables, respectively, i.e.,

$$
D_{F}=\sum_{n=1}^{N_{F}-1} D_{n} \quad D_{T}=\sum_{n=1}^{N_{L}+1} D_{n} .
$$

Based on the equivalent circuit in Fig. 3(c), the resonance frequency of the equivalent impedance $Z_{E q k}$ observed from the failed luminaire $\mathrm{Lm}_{N F}$ is calculated by the following expression:

$$
f_{r}=\frac{1}{2 \pi \sqrt{\frac{C_{E u} C_{E l}}{C_{E u}+C_{E l}} L_{c c}}} .
$$

The above expression can be rewritten considering the length of the cables as follows:

$$
f_{r,}=f_{r n} d_{F}
$$

with

$$
\begin{aligned}
& f_{r n}=\frac{1}{2 \pi \sqrt{C_{T} L_{c c}}}=\frac{1}{2 \pi \sqrt{D_{T} C_{x} L_{c c}}} \\
& d_{F}=\sqrt{\frac{1}{D_{F r}\left(1-D_{F r}\right)}} \quad D_{F r}=\frac{D_{F}}{D_{T}},
\end{aligned}
$$

where $f_{r n}$ is the resonance frequency of the complete AGL system (called natural frequency of the system) and $d_{F}$ is the resonance frequency dependence on the relative length of the cables before the failed luminaire with respect to the total length of the AGL system cables (i.e., the relative position of the failed luminaire, $D_{F r}$ ).

Note that the resonance frequency of the equivalent impedance $Z_{E q k}$ depends on the natural frequency, which is inversely proportional to the total length of the AGL cables $D_{T}$ and relative position of the failed luminaire. Fig. 4(a) illustrates the natural frequency range for typical values of the AGL system length and CCR power ratings. Typical AGL system values of the cable distributed capacitor and CCR reactance are also considered in the above plot, i.e., $C_{x}=0.126 \mu \mathrm{F}$ and $X_{c c}=5 \%\left(U_{C C R}=5 \mathrm{kV}\right)$. According to these AGL system values, this natural frequency can be in the 0.2 to $1.2 \mathrm{kHz}$ range. Note that large AGL systems and small CCR power ratings lead to low natural frequencies. Fig. 4(b) 


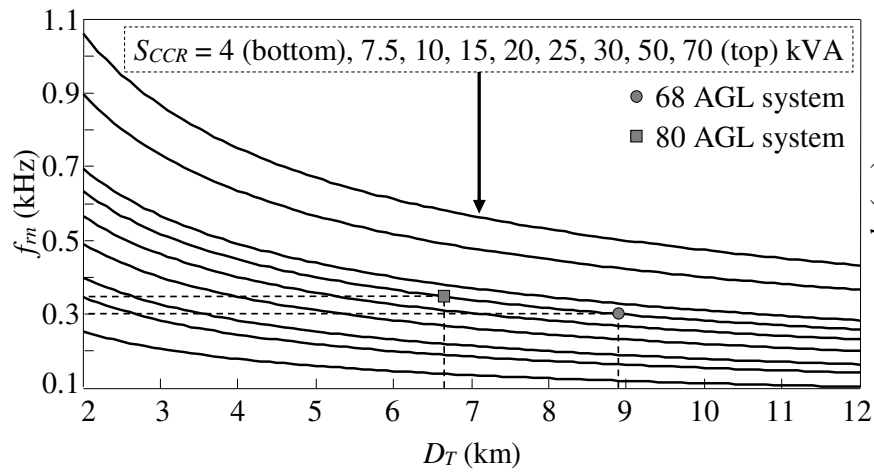

(a)

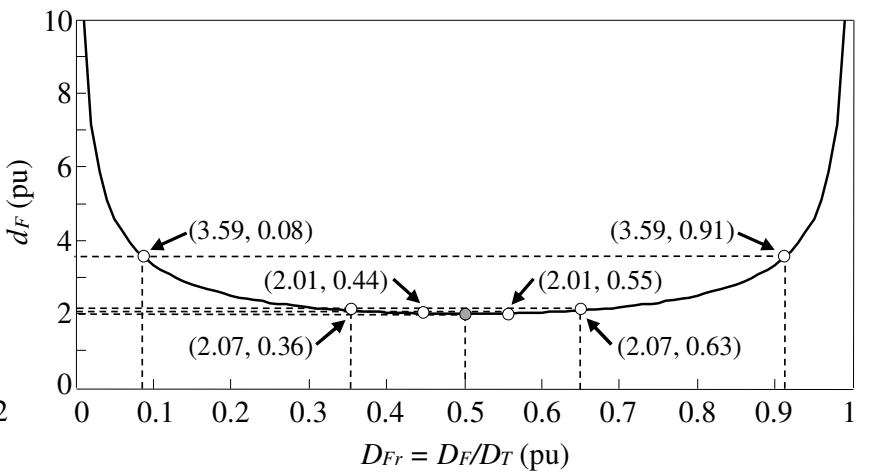

(b)

Fig. 4. Characterization of resonance frequencies. (a) Natural resonance frequency frn $\left(C_{x}=0.126 \mu \mathrm{F}\right.$ and $X_{c c}=5 \%$ with $\left.U_{C C R}=5 \mathrm{kV}\right)$, (b) Resonance frequency dependence $d_{F}$ on the relative position $D_{F r}$ of the failed luminaire.

illustrates the resonance frequency dependence on relative position of the failed luminaire. It can be observed that the lowest resonance frequency occurs when the failed luminaire is in the middle of the AGL system (grey dot at $D_{F r}=0.5 \mathrm{pu}$ ) and that this frequency is twice the natural frequency of the AGL system. Also note that failure of the first and last luminaires is not worrisome because it causes resonances at high frequencies. Expressions (5) and (6) can be a useful tool for detecting the distance at which the failure occurs, i.e., for locating the failed luminaire.

\section{FAILED LUMINAIRE LOCATION}

Luminaire failure is an important issue for safe operation of AGL systems. Thus, fast detection and location of failed luminaires become mandatory for safe operating policies. Cost-effective execution of these tasks is another challenge due to the size of AGL circuits.

\section{A. Resonance-based Procedure for Failed Luminaire Location}

The study in Section III shows that failed luminaires may be located from the resonance frequency observed from these luminaires which, according to the experimental study in Section V, could be characterized with voltage and current measurements in the CCR room. Considering this, the procedure for locating failed luminaires is as follows:

- Determine the natural frequency $f_{r n}$ from (6) and AGL system data.

- Determine the resonance frequency $f_{r}$ observed from the failed luminaire by measuring the voltage and current at CCR terminals.

- Determine the resonance frequency dependence $d_{F}$ and relative position of the failed luminaire $D_{F r}$ from (5) and (6), respectively.

- Determine the position of the failed luminaire $D_{F}$ from (6) and total length of the AGL system cables $D_{T}$.

- Locate the failed luminaire from $D_{F}$ and luminaire distribution in the AGL system.

The application of this procedure is illustrated in Section V from experimental tests made on two AGL circuits of 68 and
80 luminaires.

\section{B. Comparison with Other Procedures for Failed Luminaire Location}

In many AGL systems, failures in luminaires are detected by controlling changes in the CCR supply voltage and subsequently locating the failed luminaires by physical inspection of the AGL circuit. This involves extensive maintenance and conservation work because of the difficulty in accessing and working in this circuit [6]. Nevertheless, little research effort has been devoted to the improvement of this location procedure. Computer controlled monitoring of the AGL system for locating failed luminaires is proposed in [3] - [6]. A PLC system could be used between the luminaires and CCRs to avoid interaction of wireless technologies with radio navigation instruments and installation of new cables in AGL circuits [3], [5], [6]. PLC modems are installed in the secondary circuits of AGL transformers to detect luminaire failures and a central PLC modem is installed in the CCR room to receive and process information of failed luminaires. Data communication between luminaire PLC modems and the CCR modem occurs through the power distribution cable of the AGL system. This communication should use a narrow band frequency (from tens to a few hundreds of kilohertz) [5]. This PLC technology is still in development and therefore has drawbacks, namely cost and impact of the AGL system on communications (e.g., interference with normal AGL operations, signal attenuation between failed luminaires and CCR room, which can be as large as $80 \mathrm{~dB}$, and interactions with noise introduced into the system by the CCR). The proposed procedure is a simple, cost-effective method for location of failed luminaires. It just requires having poweranalyzer equipment in the CCR room to measure the voltage and current at CCR terminals, characterizing the resonance frequency from the ratio between the voltage and the current and locating the failed luminaire with the proposed algorithm. The main drawback of this procedure is that very accurate measurements are required for the precise location of failures. 


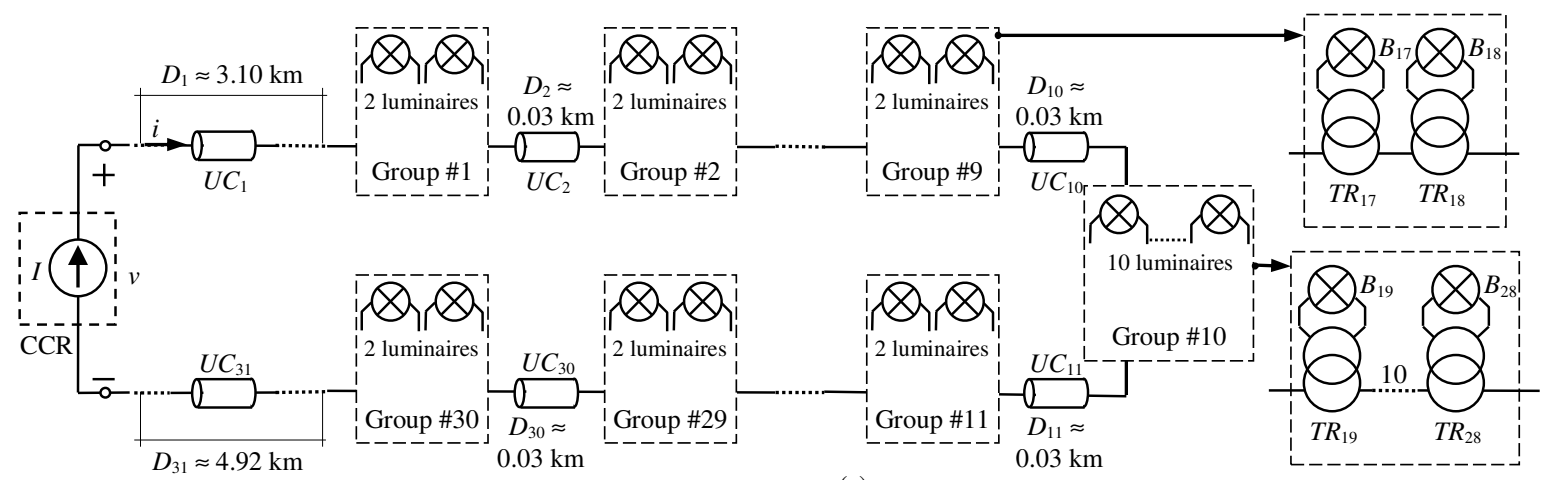

(a)

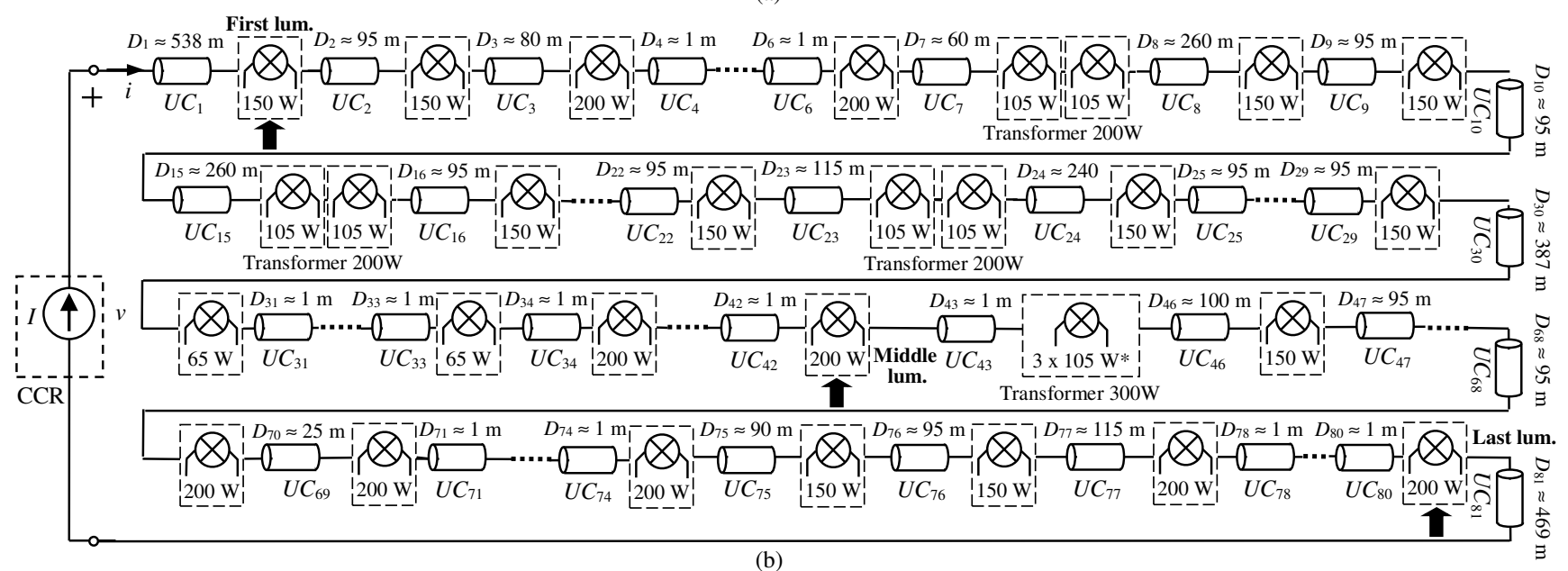

(b)

Fig. 5. AGL system applications. (a) 68 luminaire AGL system, (b) 80 luminaire AGL system.

\section{APPLICATIONS}

Field measurements were conducted on two AGL circuits of 68 and 80 luminaires at Reus airport (Catalunya, Spain) to study AGL system resonance and validate the analytical study in Section III. In order to determine the frequency response of the AGL system from a failed luminaire, a luminaire was removed and voltage $v_{F L}$ and current $i$ at the terminals of this

Table 1

AGL system parameters

\begin{tabular}{|c|c|c|c|c|c|}
\hline & Parameters & \multicolumn{4}{|c|}{68 and 80 luminaire system } \\
\hline CCR & $\begin{array}{c}\text { Model } \\
L_{c c}(\mathrm{mH})\end{array}$ & \multicolumn{4}{|c|}{$\begin{array}{c}\text { OCEM UR2000-2100 } 25 \mathrm{kVA} \\
250\end{array}$} \\
\hline $\begin{array}{l}\text { Underground } \\
\text { cable }\end{array}$ & $\begin{array}{c}R_{X}(\Omega / \mathrm{km}) \\
L_{x}(\mathrm{mH} / \mathrm{km}) \\
C_{x}(\mu \mathrm{F} / \mathrm{km})\end{array}$ & & & & \\
\hline \multirow{7}{*}{$\begin{array}{c}6.6 / 6.6 \mathrm{~A} \\
50 \mathrm{~Hz} \text { AGL } \\
\text { transformer } \\
{[13]}\end{array}$} & $P(\mathrm{~W})$ & 65 & 150 & 200 & 300 \\
\hline & $R(\Omega)$ & 0.281 & 0.281 & 0.301 & 0.411 \\
\hline & $L_{d}(\mathrm{mH})$ & 0.849 & 1.19 & 1.71 & 4.22 \\
\hline & $M_{1}(\mathrm{mH})$ & 327.6 & 693.8 & 650.0 & 663.7 \\
\hline & $M_{2}(\mathrm{mH})$ & 1.4 & 1.80 & 1.80 & 1.80 \\
\hline & $p(\mathrm{pu})$ & 1.2 & 0.906 & 1.15 & 0.905 \\
\hline & $i_{0}(\mathrm{~A})$ & 0.321 & 0.286 & 0.338 & 0.286 \\
\hline
\end{tabular}

"failed" luminaire (see AGL system in Fig. 2) were measured. Subsequently, the harmonic voltage and current values, $V_{F L, k}$ and $I_{F L, k}$ in Fig. 3(a), were calculated from these measurements. Finally, the equivalent impedance observed from the "failed" luminaire was calculated as $Z_{E q k}=V_{F L, k} / I_{F L, k}$ $(k=3,5 \ldots)$. Measurements were made with a SIGILENT SDS 102CML digital scope and numerically processed in a computer. The test results were compared with the resonance frequency in (4) and results obtained from Matlab/Simulink simulation with the AGL system equivalent circuit in Section II.B. Additional measurements of voltage $v$ and current $i$ at the CCR output terminals in Fig. 2 were performed under different operating conditions to confirm the results.

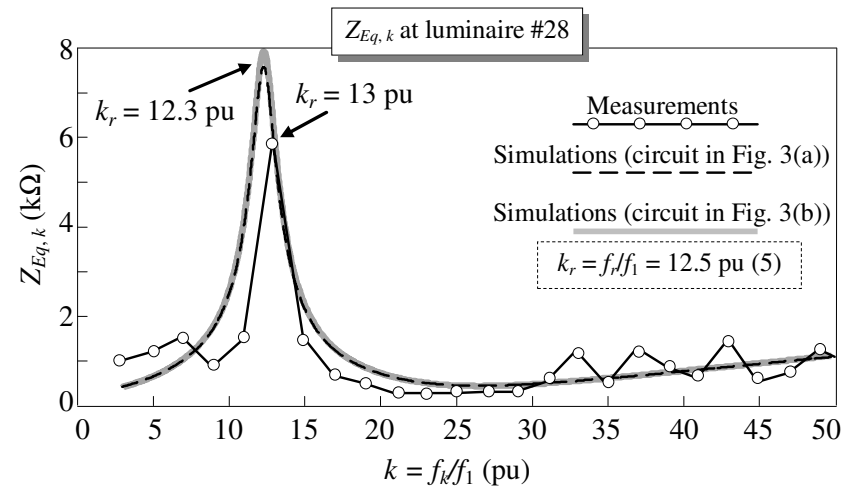

Fig. 6. Resonance test in 68 luminaire AGL system $\left(f_{1}=50 \mathrm{~Hz}\right)$. 

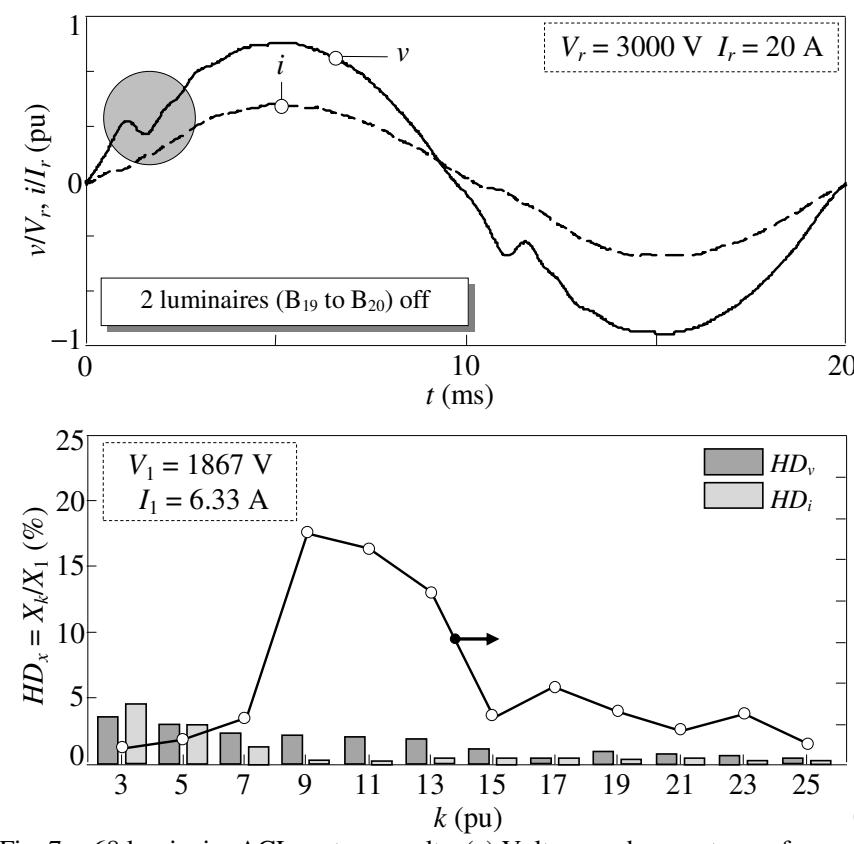

Fig. 7. 68 luminaire AGL system results. (a) Voltage and current waveforms.

\section{A. 68 Luminaire System}

Fig. 5(a) illustrates the $8.89 \mathrm{~km}$ AGL system circuit corresponding to the approach lighting system with a $25 \mathrm{kVA}$ CCR supplying 68 luminaires of $150 \mathrm{~W}$ connected through $150 \mathrm{~W}$ 6.6/6.6 A $50 \mathrm{~Hz}$ transformers (data in Table 1). In order to experimentally validate the analytical study in Section III, a luminaire of group \#10 was removed and the equivalent impedance observed from the "failed" luminaire was calculated from the above experimental procedure. Fig. 6 compares this equivalent impedance (in solid line) with those obtained from Matlab/Simulink simulations (in dotted lines) considering the circuit in Fig. 3(a). The resonance frequency obtained from the analytical expression in (5) is also labeled, and the equivalent impedance obtained from Matlab/Simulink simulation considering the approximations in Section III (i.e., circuit in Fig. 3(b)) is plotted in grey solid line to check these approximations. The results allow the approximations to be justified and show the acceptable accuracy of the analytical study.

In order to check the above resonance frequency and the influence of resonance on the AGL system, measurements for the highest brightness step and with all luminaires on and two and ten failed luminaires of group \#10 were performed. The measured voltage $\mathrm{v}$ and current $\mathrm{i}$ at the CCR output terminals with all luminaires on were $1959 \mathrm{~V}$ and $6.16 \mathrm{~A} 50 \mathrm{~Hz}$ quasisinusoidal waveforms. The measured voltage and current of the failed luminaire tests are shown in Fig. 7. Fig. 7(a) plots the measured voltage and current waveforms and Fig. 7(b) shows their harmonic spectra and the $Z_{k}=V_{k} / I_{k}$ relationship. This relationship approximately matches the equivalent impedance observed from the failed luminaires (see Fig. 6). The following observations can be made from the measurements in Fig. 7:

- In the event of luminaire failure, the transformers maintain

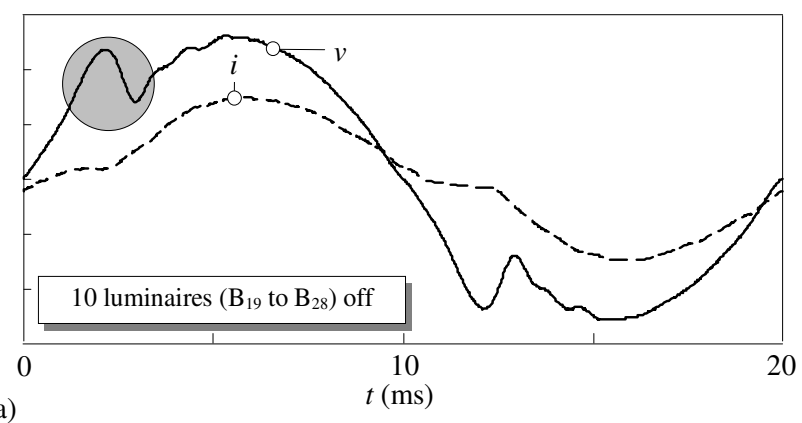

(a)

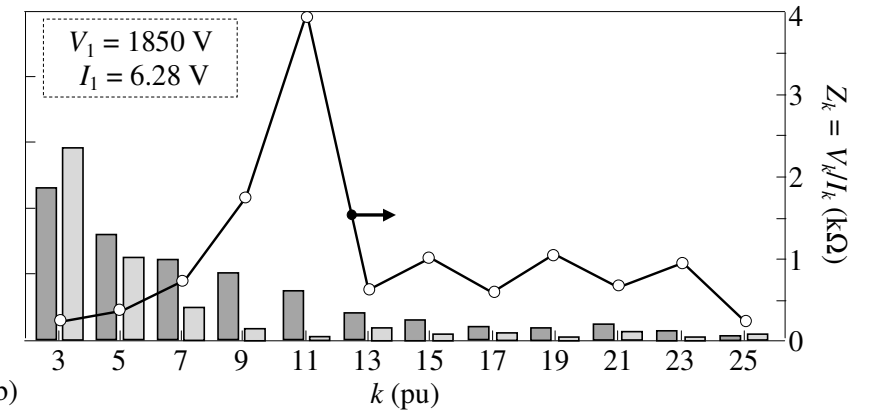

b)

circuit continuity but work in saturation because they operate in open circuit at series circuit rated current. This introduces harmonic currents into the AGL system, modifying CCR operating conditions and reducing AGL system power quality. It is concluded from the test results that luminaire failure (particularly, a large number of failed luminaires) can lead to harmonic current and voltage distortion above standard limits (e.g., IEC 6100-3-2 [16] and EN 5160 [17] limits, respectively).

- Harmonic current emissions of AGL saturated transformers are amplified around resonance frequencies in case of luminaire failure, leading to increased voltage distortion, and thus reduced power quality. This distortion can also be observed in the voltage waveform oscillations (grey circles).

- Results in the $\underline{Z}_{k}$ plotline illustrate the parallel resonance around the $9^{\text {th }}$ and $13^{\text {th }}$ harmonics. This can be observed by comparing the distortion of the $9^{\text {th }}$ to $13^{\text {th }}$ harmonic voltages (high) and currents (low). The frequency of this resonance could be determined from the voltage and current at CCR room terminals, to which the measurement equipment is usually connected. This allows location of the failed luminaire.

Failed luminaires can be located by the procedure in Section IV, as follows:

- Considering the AGL system data (see Table I), the natural frequency of the system is $300.8 \mathrm{~Hz}$ as labeled in Fig. 4(a).

- Considering the measured resonance frequency $f_{r} \approx 625 \mathrm{~Hz}$ $\left(k_{r} \approx 12.5\right.$ in Fig. 6 and Fig. 7) and the above natural frequency, $d_{F}=2.077$ and $D_{F r}=0.3646$ and 0.6353 (see Fig. 4(b)).

- From the above relative positions of the failed luminaire and total length of the AGL system, this luminaire should be located at $D_{F}=3.24$ and $5.64 \mathrm{~km}$. According to Fig. 5(a), the former value corresponds to the luminaire of group \#6. It must be noted that the flat pattern of the $d_{F}$ function 

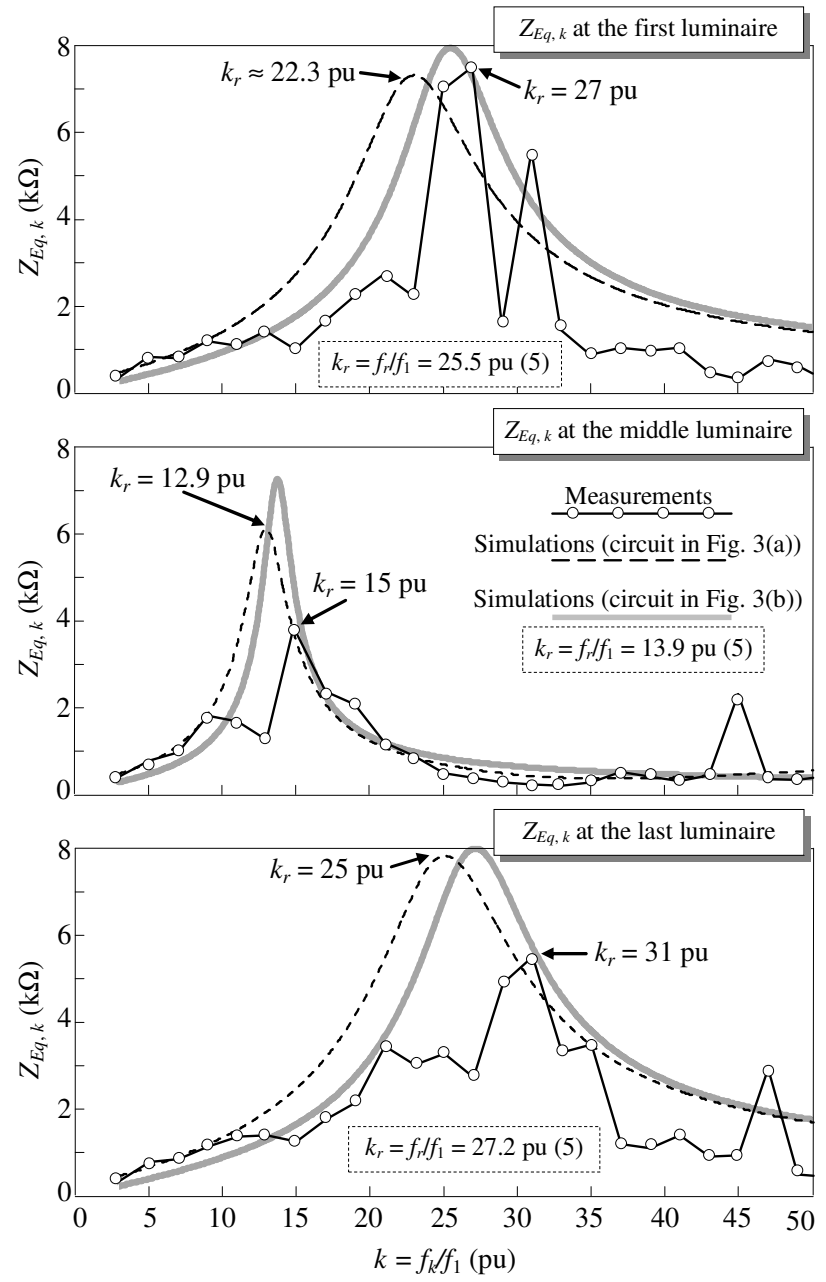

Fig. 8. Resonance test in 80 luminaire AGL system $\left(f_{1}=50 \mathrm{~Hz}\right)$.

would require a more accurate characterization of the system parameters and measurements of the resonance frequency for accurate location of the failed luminaire.

\section{B. 80 Luminaire System}

Fig. 5(b) illustrates the $6.66 \mathrm{~km}$ AGL system circuit corresponding to the runway lighting system with a $25 \mathrm{kVA}$ CCR supplying 80 luminaires of different power ratings according to their specific functions (data in Table 1):

- Runway edge luminaires: Three sets of two grounded luminaires of $105 \mathrm{~W}$ connected through a $200 \mathrm{~W}$ 6.6/6.6 A $50 \mathrm{~Hz}$ transformer and elevated luminaires of $150 \mathrm{~W}$ connected through a $150 \mathrm{~W} 6.6 / 6.6$ A $50 \mathrm{~Hz}$ transformer.

- Runway threshold luminaires: One set of three grounded luminaires of $105 \mathrm{~W}$ connected through a $300 \mathrm{~W} \mathrm{6.6/6.6} \mathrm{A}$ $50 \mathrm{~Hz}$ transformer.

- Runway threshold wingbar luminaires: Elevated luminaires of $200 \mathrm{~W}$ connected through a $200 \mathrm{~W} 6.6 / 6.6 \mathrm{~A} 50 \mathrm{~Hz}$ transformer.

- Runway end luminaires: Four grounded luminaires of $65 \mathrm{~W}$ connected through $65 \mathrm{~W}$ 6.6/6.6 A $50 \mathrm{~Hz}$ transformers.

In order to experimentally validate the analytical study in Section III, the first, middle and last luminaires (labeled with black arrows in Fig. 5(b)) were removed and the equivalent impedances observed from these "failed" luminaires were calculated by the previous experimental procedure. Fig. 8 compares these equivalent impedances (in solid lines) with the Matlab/Simulink simulation ones (in dotted lines) considering the circuit in Fig. 3(a). The resonance frequencies obtained from the analytical expression in (5) are also labeled. The equivalent impedances obtained from Matlab/Simulink simulations considering the approximations in Section III (i.e., the circuit in Fig. 3(b)) are also plotted in grey solid lines to check these approximations. Similar conclusions to those drawn for the 68 luminaire AGL system are obtained.

In order to check the above resonance frequencies and influence of resonance on the AGL system, measurements of voltage $v$ and current $i$ at the CCR output terminals for the highest brightness step and with all luminaires on and the first, middle and last failed luminaires were also performed. The measured voltage $v$ and current $i$ at the CCR output terminals with all luminaires on were $2300 \mathrm{~V}$ and $6.43 \mathrm{~A} 50 \mathrm{~Hz}$ quasisinusoidal waveforms. The measured voltage and current of the failed luminaire tests are shown in Fig. 9. Fig. 9(a) plots the measured voltage and current waveforms and Fig. 9(b) shows their harmonic spectra and the $\underline{Z}_{k}=V_{k} / I_{k}$ relationship. The following observations can be made from the measurements in Fig. 9:

- Resonances amplify the harmonic current emissions of AGL saturated transformers, leading to increased voltage distortion around the resonance frequency. This can also be observed in the voltage waveform oscillations (grey circles).

- Results in the $\underline{Z}_{k}$ plotlines illustrate the parallel resonance around the $23^{\mathrm{rd}}$ and $27^{\text {th }}$ harmonics for the first luminaire, the $13^{\text {th }}$ and $15^{\text {th }}$ harmonics for the middle luminaire and the $25^{\text {th }}$ and $27^{\text {th }}$ harmonics for the last luminaire. These results confirm the results in Fig. 4(b) as failure of the middle luminaire leads to lower order harmonic resonances than failure of the first and last luminaires.

- Results in the $\underline{Z}_{k}$ plotlines confirm that resonance frequency may be determined from voltage and current measurements at CCR room terminals (i.e., failed luminaires may be located from these measurements).

The failed luminaire can be located by the procedure in Section IV, as follows:

- Considering the AGL system data (see Table 1), the natural frequency of the system is $347.5 \mathrm{~Hz}$ as labeled in Fig. 4(a).

- Considering the measured resonance frequencies $f_{r} \approx 1250 \mathrm{~Hz}$ (first and last luminaires) and $700 \mathrm{~Hz}$ (middle luminaire) and the above natural frequency, $d_{F}=3.596$ and $D_{F r}=0.084$ and 0.915 for the first and last luminaires and $d_{F}=2.013$ and $D_{F r}=0.441$ and 0.558 for the middle luminaire (see Fig. 4(b)).

- From the above relative positions of the failed luminaires and total length of the AGL system, these luminaires are located at $D_{F}=0.562$ and $6.096 \mathrm{~km}$ and $D_{F}=2.93$ and 3.71 $\mathrm{km}$. According with Fig. 5(b), the value $0.562 \mathrm{~km}$ corresponds to the first luminaire, the value $6.069 \mathrm{~km}$ corresponds to the third luminaire from the end and the values 2.93 and $3.71 \mathrm{~km}$ correspond to the middle 

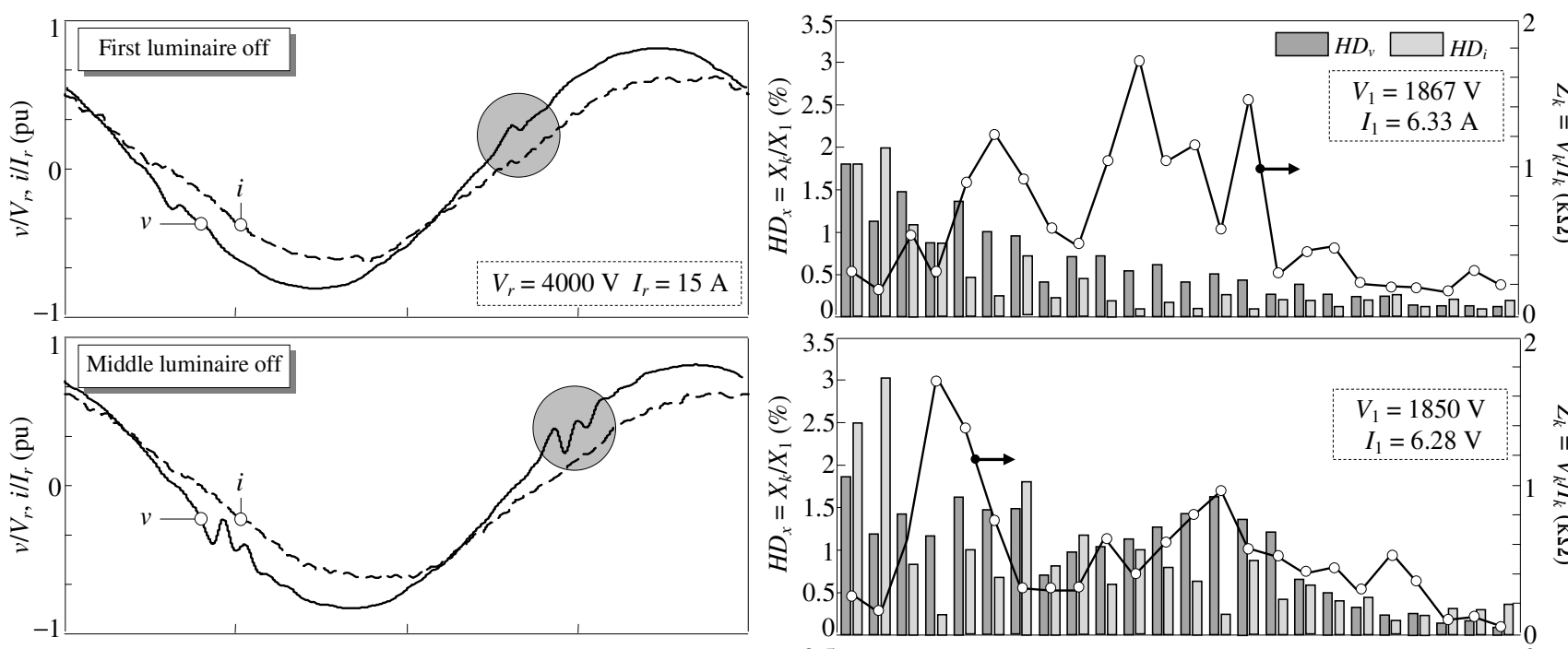

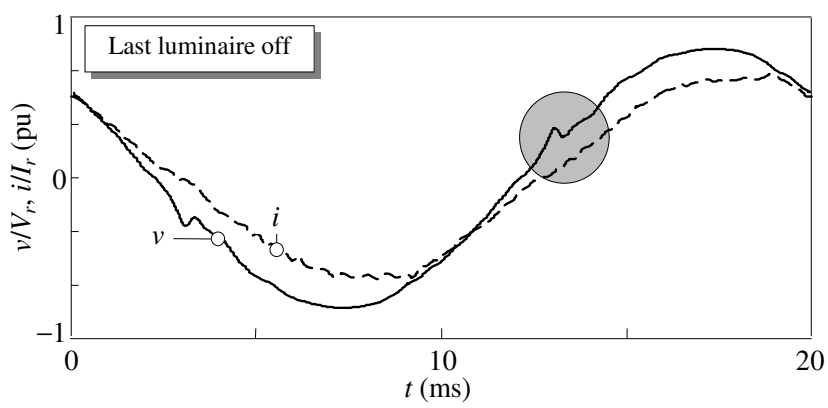

(a)

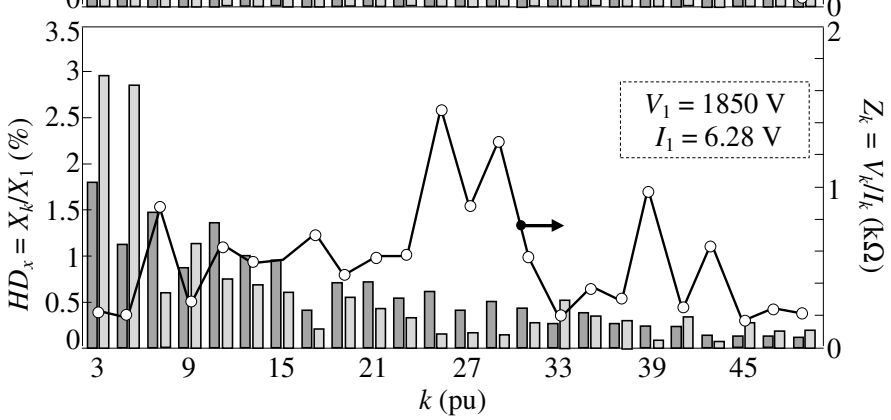

(b)

Fig. 9. 80 luminaire AGL system results. (a) Voltage and current waveforms. (b) Voltage and current harmonic spectra and equivalent impedance plotline.

luminaire, which is placed at $3.63 \mathrm{~km}$.

\section{CONCLUSIONS}

The paper studies the parallel resonance phenomenon in AGL systems and presents analytical expressions to determine resonance frequency at failed luminaire terminals, which mainly depends on the CCR transformer inductance and underground cable capacitors. Resonance frequency can be expressed in function of the natural frequency of the AGL system and position of failed luminaires. Based on this, a procedure for locating failed luminaires from measurements of the parallel resonance at CCR room terminals is proposed. The accuracy of the proposed procedure depends on the precise characterization of system parameters and measurements of resonance frequency. Matlab/Simulink simulations with the AGL system equivalent circuit and field measurements of 68 and 80 luminaire AGL systems at Reus airport are presented to validate the goodness of the analytical expressions and failed luminaire location procedure.

\section{REFERENCES}

[1] Aerodrome design manual, Part 4: Visual aids, $4^{\text {th }}$ Edition International Civil Aviation Organization, 2004.

[2] Aerodromes, Volume I: Aerodrome design and operations, Annex 14 to the convention on International Civili Aviation, $4^{\text {th }}$ Edition International Civil Aviation Organization, 2004.

[3] Saraf, N., Salvi, R., Salunkhe, N., and R. Sahasrabuddhe. "Airfield lamp monitoring \& control systems," Int. Conf. on Information,
Communication and Embedded Systems (ICICES 2013), Febr. 2013, pp. 1141-1143.

[4] Kevin, P. E. Integration of aviation lighting system and computer controlled monitoring system. IEEE Int. Conf. on Systems, Man and Cybernetics, Oct. 1996, 1132-1137.

[5] Granado, J., Chavez, J., Torralba, A., and Oria, A. C. Modeling airfield lighting systems for narrowband power-line communications. IEEE Trans. on Power Delivery, 25, 4 (Oct. 2010), 2399-2405.

[6] Bing-Yuan, W., Li-Wen, W. Remote automatic monitor system for airfield lightning. Int. Conf. on Computer Applications and System Modeling (ICCASM 2010), 2010, Vol. 8, 542-545.

[7] Giardini, D., and Galloway, J. H. A single phase matrix down-converter for airport lighting regulation. $34^{\text {th }}$ IAS Annual Meeting Industry Applications, Oct. 2011, vol. 2, 1153-1156.

[8] Freschi, F., Mitolo, M., and Tommasini, R. Electrical safety of aeronautical ground lighting systems. IEEE Trans. on Industry Applications, 51, 3 (May-June 2015), 2003-2008.

[9] Sheng, C., Wenying, Y., and Guoufu, Z. Reliability analysis of airport lighting aid system based on light source failure. Int. Conf. on Electrical Contacts, May 2012, 475-478.

[10] OCEM AirfieldTechnology, "Constant current regulators. New generation", EnergyTechnology.

[11] Smith, C. C. Some aspects of airport lighting. Electronics and Power, 16, 3 (1970), 85-89.

[12] Bejleri, M., Rakov, V. A., Uman, M. A., Rambo, K. J., Mata , C. T., and Fernandez, M. I. Triggered lighting testing of an airport runway lighting system. IEEE Trans. on Electromagnetic Compatibility, 46, 1 (Feb. 2004), 96-101.

[13] Vidal, D., Monjo, Ll., Sainz, L., and Pedra, J. Model of aeronautical ground lighting system transformer. IET Electric Power Applications, 9, 3 (March 2015), 239-247.

[14] Vidal, D., Monjo, Ll., Sainz, L., and Pedra, J. Aeronautical ground lighting system study: field measurement and simulations. IET Generation, Transmission and Distribution, accepted for publication doi: 10.1049/iet-gtd.2015.1536. 
[15] MAtLAB 7.9 (R2009b) and Simulink, The MathWorks, Natick, MA: 2009.

[16] IEC Electromagnetic Compatibility (EMC), Part 3: Limits, Section 2: Limits for harmonics current emissions (equivalent input current $<16 \mathrm{~A}$ per phase), IEC-61000-3-2, Ed. 3, 2005.

[17] EN 50160, Voltage characteristics of electricity supplied by public electricity networks, 2011.

[18]

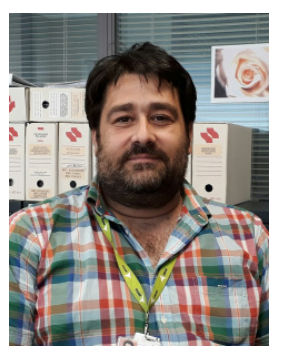

Daniel Vidal was born in Tortosa (Spain) in 1976. $\mathrm{He}$ received his B.S. degree in Electrical Engineering and his M.Eng. in Air-Conditioning Technologies and Energy Efficiency in Buildings from the Universitat Rovira i Virgili Spain, in 2004 and 2012, respectively. He is currently working in airport maintenance systems at Reus airport (Catalunya, Spain) and pursuing the Ph.D. degree in power quality at aeronautical electrical installations. His area of interest includes airport electrical circuits and beacon systems.

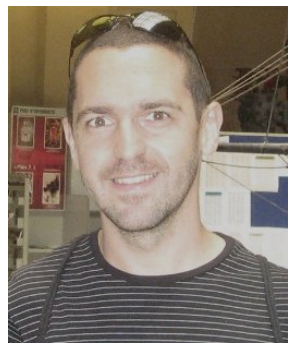

Lluís Monjo was born in Tremp, Spain, in 1983. $\mathrm{He}$ received the B.S. degree in electrical engineering, the M.Eng. degree in energy and the Ph.D. from the Universitat Politècnica de Catalunya (UPC), Barcelona, Spain, in 2007, 2009 and 2013 respectively. He has been professor with the Electrical Engineering Department of the UPC since 2007 to 2015. Since 2016 he is Assistant Professor in the Department of Industrial Systems and Design in the Universitat Jaume I (UJI) in Castelló de la Plana. His research interests include electric machines, renewable generation, and power system quality.

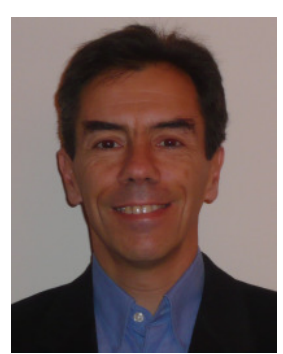

Luis Sainz was born in Barcelona (Spain). He received his M.S. degree in Industrial Engineering and his Ph. D. degree in Engineering from the Universitat Politècnica de Catalunya (UPC), Barcelona, Spain, in 1990 and 1995 respectively. Since 1991, he is professor in the Department of Electrical Engineering of the UPC. His research interest lies in the area of power quality and gridconnected VSCs. 